Article

\title{
Local Polynomial Smoother for Solving Bagley-Torvik Fractional Differential Equations
}

\author{
Tianshun Yan $^{1}$, Shuanghua Luo ${ }^{2}$
}

\begin{abstract}
Local polynomial smoother (LPS) is a weighted local least-squares nonparametric method. It provides a local Taylor series fit of the data at any location and can be directly used in a differential equation to provide a numerical scheme. In this article, we introduce this new nonparametric idea based on local polynomial smoother, for acquiring the numerical solution of the Bagley-Torvik fractional-order differential equations. Furthermore, this paper will present a numerical comparison with some methods, such as legendre operational matrix and pseudo-spectral method. The efficiency and accuracy of the LPS method are demonstrated by two numerical examples.
\end{abstract}

Keywords: numerical solution; · Bagley-Torvik fractional differential equations; · local polynomial smoother

1.School of Mathematics and Statistics, Xi'an Jiaotong University, Xi'an 710049, China

Corresponding author Email: yantianshun@yahoo.cn or adsyan731868@stu.xjtu.edu.cn 2. School of Science, Xi'an Polytechnic University, Xi'an 710048, China

Email: rabbitdoctor@outlook.com 


\section{Introduction}

The fractional differential equations appear more and more frequently in different research areas and engineering applications. Phenomena in electromagnetics, acoustics, viscoelasticity, and electrochemistry and material science are also described by differential equations of fractional order. The solution of the differential equation containing fractional derivative is much involved. For example, fractional-order derivatives have been successfully used to model damping forces with memory effect or to describe state feedback controllers [1-4]. Consequently, an effective and easy to use method for solving such equations is needed. it was undoubtedly the book written by Oldham and Spanier [3] which played an outstanding role in the development of this subject.

The Bagley-Torvik equation with 1/2-order derivative or 3/2-order derivative describes motion of real physical systems, an immersed plate in a Newtonian fluid and a gas in a fluid, respectively $[1,2,4,5]$. The Bagley-Torvik equation has been numerically solved by using, Adomian decomposition method [6], the generalized Taylor collocation method [7], the fractional linear multistep methods, a predictor-corrector method of Adams type [8, 9], Haar wavelet operational matrix [10]. Besides, during the last decades, several methods have been employed to solve fractional differential equations. Diethelm et al. [12] and Ford et al. [13] have reviewed some of the existing methods and explained their respective strengths and weaknesses. There are some further methods, such as operational method [14], homotopy analysis method [15], differential transform method [16-18] and other methods [11,19-23].

Recently, Su and Yan [24], have used local polynomial smoother method for the numerical solution of integro-differential equations. Later, Su and Yan [25, 26], manage to solve ordinary differential equations and partial differential equations respectively by using this method. Numerical results demonstrate that local polynomial fitting method is more accurate, simply and efficient. In this study, we consider the following initial value problem in the case of the inhomogeneous Bagley-Torvik equation, then compared with other methods:

$$
P(t) D^{2} y(t)+R(t) D^{\frac{3}{2}} y(t)+S(t) y(t)=f(t), 0 \leq t \leq b
$$

with the boundary conditions:

$$
y(a)=\gamma_{0}, y(b)=\gamma_{1}
$$

where $y^{(0)}(t)=y(t)$ and $y(t)$ is an unknown function, $P(t), R(t), S(t)$, and $f(t)$ are functions defined on the interval $a \leq t \leq b$, and $\mathrm{b}, \gamma_{0}$ and $\gamma_{1}$ are real constants. The 
main destination of this paper is to explore a fast algorithm for numerical solution of Bagley-Torvik FDEs (1) using a new nonparametric method, local polynomial smoother (LPS). In Section 2, we present the definition of fractional-order integration and fractional-order differentiation. In Section 3, local polynomial smoother method are described and their main thinking are reviewed. In Section 4, three parameters selections, the kernel $K$ and the bandwidth $h$, are discussed in LPS method. In Section 5 , we employ local polynomial Smoother method for solving of the Bagley-Torvik FDEs (1). Finally, in Section 7, we present some numerical tests to validate the theoretical results and we make comparisons of the methods discussed in the article.

\section{Basic definitions}

We first give the definition of fractional-order integration and fractional-order differentiation [17]. For the concept of fractional derivative, we will adopt Caputos definition, which is a modification of the Riemann-Liouville definition and has the advantage of dealing properly with initial value problems.

Definition 2.1 A real function $f(x), x>0$, is said to be in the space $C_{\mu}, \mu \in R$ if there exists a real number $p>\mu$ such that $f(x)=x^{p} f_{1}(x)$, where $f_{1}(x) \in C[0, \infty]$. Clearly, $C_{\mu}<C_{\beta}$ if $\beta<\mu$.

Definition 2.2 A function $f(x), x>0$, is said to be in the space $C_{\mu}^{M}, \mu, m \in N \cup\{0\}$ if $f^{(m)} \in C_{\mu}$.

Definition 2.3 The Riemann-Liouville fractional integral operator of order $\alpha \geq 0$ of a function, $f \in C_{\mu}, \mu \geq-1$, is defined as

$$
\begin{aligned}
& J^{\alpha} f(x)=\frac{1}{\Gamma(\alpha)} \int_{0}^{x}(x-t)^{\alpha-1} f(t) d t, \quad \alpha \geq 0, \quad x>0 \\
& J^{0} f(x)=f(x) .
\end{aligned}
$$

The properties of the operator $J^{\alpha}$ can be found in $[34,35]$; we mention only the following:

For $f \in C_{\mu}, \mu \geq-1, \alpha, \beta \geq 0$ and $\gamma>-1$ :

$$
\begin{aligned}
& 1^{0}: J^{\alpha} J^{\beta} f(x)=J^{\alpha+\beta} f(x), \\
& 2^{0}: J^{\alpha} J^{\beta} f(x)=J^{\alpha+\beta} f(x), \\
& 3^{0}: J^{\alpha} x^{\gamma}=\frac{\Gamma(\gamma+1)}{\Gamma(\alpha+\gamma+1)} x^{\alpha+\gamma} .
\end{aligned}
$$


The Riemann-Liouville derivative has certain disadvantages when trying to model real-world phenomena using fractional differential equations. Therefore, we will introduce a modified fractional differential operator $D_{*}^{\alpha}$ proposed by Caputos work on the theory of viscoelasticity [39].

\section{Local polynomial smoother}

Local polynomial smoother technique was first proposed by Fan.J [27-29]. And, this method was also mentioned and used by $\mathrm{Su}[30]$. In order to describe the basic ideas of the LPS, firstly, we introduce the mathematical thoughts of local polynomial smoother. This idea were detailed mentioned in [27-29]. The basic idea is to form a locally optimal Taylor series to the data by minimizing the $L_{2}$ norm of the residual between the polynomial model and the data. Since the form of Smoother function is not specified, so the data points with long distance from $t_{0}$ provides little information to $y\left(t_{0}\right)$. Therefore, we can only use the local data points around $t_{0}$. We suppose that $y(t)$ has $p+1$ derivative at $t_{0}$, by the Taylor expansion, for point $t$, located in the neighborhood of this point $t_{0}$, we can use the $p$-order multivariate polynomials to locally approximate $y(t)$, and the surrounding local point of $t_{0}$, we model $y(t)$ as Taylor series expansion around any pint $t_{0}$ is:

$$
y(t) \approx \sum_{j=0}^{p} \beta_{j}\left(t-t_{0}\right)^{j} .
$$

where parameter $\beta_{j}$ depends on $t_{0}$, so called local parameter. Obviously, the local parameter $\beta_{j}=y^{(j)}\left(t_{0}\right) / j$ ! fits the local model with local data and it can be minimized,

$$
\sum_{i=1}^{n}\left[Y_{i}-\sum_{j=0}^{p} \beta_{j}\left(t_{i}-t_{0}\right)^{j}\right]^{2} K\left(\frac{t_{i}-t_{0}}{h}\right)
$$

where $h$ controls the size of the bandwidth of local area. Using matrix notation to represent the local polynomial smoother is more convenient. Below is the design matrix corresponding to (3) with $t$ and $Y$ :

$$
X=\left(\begin{array}{cccc}
1 & t_{1}-t_{0} & \cdots & \left(t_{1}-t_{0}\right)^{p} \\
\vdots & \vdots & \ddots & \vdots \\
1 & t_{n}-t_{0} & \cdots & \left(t_{n}-t_{0}\right)^{p}
\end{array}\right), Y=\left(\begin{array}{c}
Y_{1} \\
Y_{2} \\
\vdots \\
Y_{n}
\end{array}\right)
$$


The weighted least squares problem (3) can be written as

$$
\min (Y-X \beta)^{T} W(Y-X \beta),
$$

here,

$$
W=\operatorname{diag}\left(K_{h}\left(t_{1}-t_{0}\right), \cdots, K_{h}\left(t_{n}-t_{0}\right)\right), K_{h}=\frac{1}{h} K\left(\frac{\dot{h}}{h}\right)
$$

so the solution vector is

$$
\beta=\left(X^{T} W X\right)^{-1} X^{T} W Y .
$$

Furthermore, we can get the estimation $\hat{y}\left(t_{0}\right)$,

$$
\hat{y}\left(t_{0}\right)=e_{1}\left(X^{T} W X\right)^{-1} X^{T} W Y,
$$

where $e_{1}$ is a column vector (the same size of $\beta$ ) with the first element equal to 1 , and the rest equal to zero, that is, $e_{1}=(1,0, \cdots, 0)_{1 \times(p+1)}$. The selection of $K$ does not influence the results much. We selected the quartic kernel as follows

$$
K(u)= \begin{cases}\frac{3}{4}\left(1-u^{2}\right)_{+}, & \text {if }|u| \leq 1 \\ 0, & \text { otherwise }\end{cases}
$$

\section{LPS for Bagley-Torvik equation}

In this section, the LPS method for solving (1) is outlined. As we know, datas $Y_{i}$ at $t=t_{i}$ in fractional differential equation are unknown, consequently, instead of estimating data at a specific point $t_{i}$, we want to find a best match of some unknown data $Y_{i}, i=1,2, \ldots$.We could vary Taylor series residual density (4) at $t_{i}$ with respect to these unknown data. Let equation(8) be an approximate solution of (1):

$$
y(t) \approx \sum_{j=0}^{p} \beta_{j}\left(t-t_{0}\right)^{j} .
$$

where, $t_{1}=a<t_{2}<\cdots<t_{n}=b$. The conventional collocation method can be expressed $t_{i}=a+\frac{b-a}{n-1}(i-1), \quad i=1, \cdots, n$. We can adjust the value of parameter $n$ flexibly, for example $n=30,50,70$. It is required that the approximate solution (8) 
satisfies the (1-2) at the point $t=t_{i}$. Putting expression (8) into equation (1), we can obtain expression as follows:

$$
\begin{aligned}
& P(t) \cdot \sum_{j=2}^{p} j(j-1) \beta_{j}\left(t-t_{0}\right)^{j-2}+R(t) \frac{1}{\Gamma\left(2-\frac{3}{2}\right)} \int_{0}^{t}(t-s)^{2-\frac{3}{2}-1} . \\
& \sum_{j=2}^{p} j(j-1) \beta_{j}\left(s-t_{0}\right)^{j-2} d s+S(t) \sum_{j=0}^{p} \beta_{j}\left(t-t_{0}\right)^{j} \approx f(t),
\end{aligned}
$$

In order to satisfy unique solution theorem of integral-differential equation, we should take initial conditions (2) into account. Consequently, putting (8) to initial conditions (2), then we can acquire the approximate expression (11) as follows:

$$
\sum_{j=0}^{p} \beta_{j}\left(a-t_{0}\right)^{j} \approx \gamma_{0}, \quad \sum_{j=0}^{p} \beta_{j}\left(b-t_{0}\right)^{j} \approx \gamma_{1}
$$

Consequently, according to the deduction process of equation (4), we can obtain easily the minimization function of problems (1-2), that is to say, the minimization function (Taylor series residual density) can be varied as (11):

$$
\begin{aligned}
& {\left[\gamma_{0}-\sum_{j=0}^{p} \beta_{j}\left(a-t_{0}\right)^{j}\right]^{2} \cdot K_{h}\left(0-t_{0}\right)^{j}+\sum_{i=2}^{p}\left\{f\left(t_{i}\right)-P\left(t_{i}\right) \cdot\right.} \\
& \sum_{j=2}^{p} j(j-1) \beta_{j}\left(t_{i}-t_{0}\right)^{j-2}-R\left(t_{i}\right) \frac{1}{\Gamma\left(2-\frac{3}{2}\right)} \int_{0}^{t_{i}}\left[\left(t_{i}-s\right)^{2-\frac{3}{2}-1} .\right. \\
& \left.\left.\sum_{j=2}^{p} j(j-1) \beta_{j}\left(s-t_{0}\right)^{j-2}\right] d s-S\left(t_{i}\right) \sum_{j=0}^{p} \beta_{j}\left(t-t_{0}\right)^{j}\right\} \cdot K_{h}\left(t_{i}-t_{0}\right)^{j} \\
& +\left[\gamma_{1}-\sum_{j=0}^{p} \beta_{j}\left(1-t_{0}\right)^{j}\right]^{2} \cdot K_{h}\left(b-t_{0}\right)^{j}
\end{aligned}
$$


Combination equations (9), (10) with (11), we can acquire the system which can be expressed (12):

$$
\left\{\begin{array}{c}
i=1, \quad A_{i, j_{1}}=\left(a-t_{0}\right)^{j_{1}} \quad j_{1}=0, \cdots, p \quad y(1)=\gamma_{0}, \\
i=2, \cdots, n-1, \quad B_{i, j_{2}}=P\left(t_{i}\right) \cdot j_{2}\left(j_{2}-1\right)\left(t_{i}-t_{0}\right)^{j_{2}-2} \quad j_{2}=2, \cdots, p \\
C_{i, j_{3}}=R\left(t_{i}\right) \frac{1}{\Gamma\left(2-\frac{3}{2}\right)} \int_{0}^{t_{i}}\left[\left(t_{i}-s\right)^{2-\frac{3}{2}-1} \cdot j_{3}\left(j_{3}-1\right) .\right. \\
\left.\beta_{j_{3}}\left(s-t_{0}\right)^{j_{3}-2}\right] d s, \quad j_{3}=2, \cdots, p \\
p \\
\left.D_{i, j_{4}}=S\left(t_{i}\right) \sum_{j_{4}=0}^{p} \beta_{j}\left(t_{i}-t_{0}\right)^{j_{4}}\right\} \quad j_{4}=0, \cdots, p \\
y(i)=f\left(t_{i}\right), \quad \\
i=n, \quad G_{i, j_{5}}=\left(b-t_{0}\right)^{j} \quad j_{5}=0, \cdots, p \quad y(n)=\gamma_{1},
\end{array}\right.
$$

then, the matrix form(5) can be written as follows by using (13-14).

$\chi=\left[\begin{array}{ccc}A_{10} & A_{11} & A_{12} \\ D_{20} & D_{21} & B_{22}+C_{22}+D_{22} \\ D_{30} & D_{31} & B_{33}+C_{33}+D_{33} \\ \vdots & \vdots & \ddots \\ \vdots & \vdots & \\ \vdots & \vdots & \\ D_{n-1,0} & D_{n-1,1} & B_{n-1,3}+C_{n-1,3}+D_{n-1,3} \\ G_{n 0} & G_{n 1} & G_{n 2}\end{array}\right.$

$$
\Upsilon=\left[\begin{array}{c}
y(1) \\
y(2) \\
\vdots \\
y(n-1) \\
y(n)
\end{array}\right]=\left[\begin{array}{c}
\gamma_{0} \\
f\left(t_{i}\right) \\
\vdots \\
f\left(t_{n-1}\right) \\
\gamma_{n}
\end{array}\right]
$$

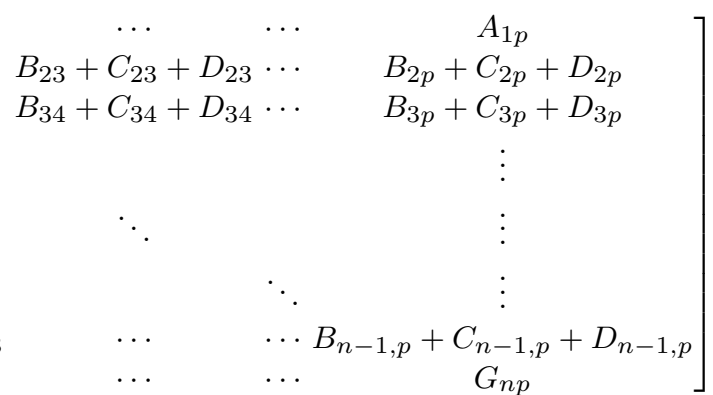

(13) 


$$
\varpi=\left[\begin{array}{lrrrr}
K_{h}(a-x) & & & \\
& K_{h}\left(X_{2}-x\right) & & & \\
& & & & \\
& & & & \\
& & K_{h}\left(X_{3}-x\right) & & \\
& & \ddots & & \\
& & & K_{h}\left(X_{4}-x\right) & \\
& & & & K_{h}(b-x)
\end{array}\right]
$$

where

$$
K_{h}=\frac{1}{h} K\left(\frac{\dot{h}}{h}\right)
$$

Putting (14-15) to (6),

$$
\hat{y}\left(t_{0}\right)=e_{1}\left(\chi^{T} \varpi \chi\right)^{-1} \chi^{T} \varpi \Upsilon
$$

where $e_{1}=(1,0,0, \cdots, 0)^{T}{ }_{p+1,1}$. In this paper, we choose the optimal weight function

$$
K(u)= \begin{cases}\frac{3}{4}\left(1-u^{2}\right)_{+}, & \text {if } \quad|u| \leq 1 \\ 0, & \text { otherwise }\end{cases}
$$

Then estimated set of coefficients $\beta_{i}$ can be obtained by solving matrix system solution, therefore, approximate-value $\hat{y}\left(t_{0}\right)$ at point $t_{0}$ can be obtained. Repeating the above steps, we can acquire $\hat{y}\left(t_{i}\right)$ at point $t_{i}, i=1, \cdots, n$, where $n$ can be selected any positive integer.

\section{Numerical examples}

In this section, in order to illustrate the description above and to show the efficiency of the mentioned method for solving equation (1-2), we give the values of the exact solution $y(t)$, In tables and figures, the approximate solution $\hat{y}(t)$, the absolute error function $e_{A E}(t)=|y(t)-\hat{y}(t)|$ at the selected points of the given interval. We have performed all numerical computations in MATLAB.

Example 1 ([34]) First, we consider the Bagley-Torvik equation

$$
y^{(2)}(t)+y^{(3 / 2)}(t)+y(t)=1+t, \quad 0 \leq t \leq 1
$$


with the boundary conditions $y(0)=1$ and $y(1)=2$, and the exact solution $y(t)=1+t$, certainly, $P(t)=R(t)=S(t)=1, f(t)=1+t$. To simplify the procedures, giving $n=11$, $p=1, h=0.5$, we calculate the estimator $\hat{y}\left(t_{i}\right) \mathrm{i}=1,2, \ldots, 11$ at point $t=t_{i}$, such as $\hat{y}\left(t_{0}\right)$

$$
\begin{aligned}
& \hat{y}\left(t_{0}\right)=e_{1}\left(\chi^{T} \varpi \chi\right)^{-1} \chi^{T} \varpi \Upsilon, \\
& \chi=\left[\begin{array}{cccc}
1 & -1 & 1 & -1 \\
1 & -0.9 & 5.052 & -12.407 \\
1 & -0.8 & 5.8107 & -13.556 \\
1 & -0.7 & 6.3733 & -13.863 \\
1 & -0.6 & 6.844 & -13.681 \\
1 & -0.5 & 7.2633 & -13.152 \\
1 & -0.4 & 7.6517 & -12.349 \\
1 & -0.3 & 8.0218 & -11.318 \\
1 & -0.2 & 8.3813 & -10.806 \\
1 & -0.1 & 8.736 & -8.6722 \\
1 & 0 & 0 & 0
\end{array}\right]
\end{aligned}
$$

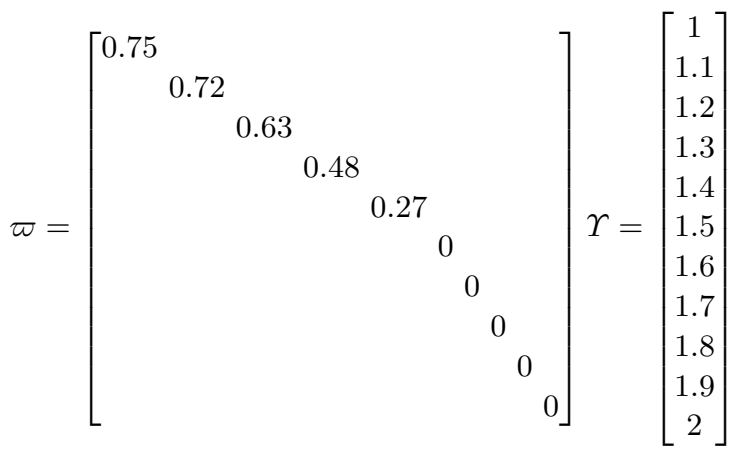

Fig 1 shows local polynomial smoother for example 1 given parameters $n=51$, $p=0, h=0.1$.

Example $2([35])$

Let us consider Bagley-Torvik equation

$$
y^{(3 / 2)}(x)+y(x)=\frac{2 x^{1 / 2}}{\Gamma(3 / 2)}+x^{2}-x, 0 \leq x \leq 1
$$


10

Table 1 Comparison of the absolutes for the numerical results and absolute errors at point $t_{i}=0.1: 0.1: 1.0$ for Example 1.

\begin{tabular}{cccc}
\hline \hline \multicolumn{2}{c}{$\begin{array}{c}\text { Absolute errors } \\
t_{i} \\
\text { for } n=50, p=1, h=0.2 \\
\text { LPS method }\end{array}$} & $\begin{array}{c}\text { Absolute errors } \\
\text { for } n=100, p=1, \mathrm{~h}=0.15 \\
\text { LPS method }\end{array}$ & $\begin{array}{c}\text { Absolute errors } \\
\text { for } \mathrm{m}=8 \alpha=0.85 \text { with } \\
\text { Legendre operational matrix [32] }\end{array}$ \\
\hline \hline 0.1 & $2.2 \times 10^{-11}$ & $6.7 \times 10^{-13}$ & $8.0 \times 10^{-4}$ \\
0.2 & $4.0 \times 10^{-11}$ & $1.2 \times 10^{-12}$ & $1.2 \times 10^{-3}$ \\
0.3 & $2.5 \times 10^{-12}$ & $1.5 \times 10^{-15}$ & $6.6 \times 10^{-4}$ \\
0.4 & $4.6 \times 10^{-13}$ & $5.3 \times 10^{-15}$ & $8.0 \times 10^{-4}$ \\
0.5 & $2.0 \times 10^{-13}$ & $3.0 \times 10^{-16}$ & $7.5 \times 10^{-4}$ \\
0.6 & $1.3 \times 10^{-12}$ & $5.2 \times 10^{-14}$ & $5.9 \times 10^{-4}$ \\
0.7 & $5.2 \times 10^{-12}$ & $6.0 \times 10^{-12}$ & $7.6 \times 10^{-4}$ \\
0.8 & $6.8 \times 10^{-10}$ & $3.7 \times 10^{-14}$ & $1.8 \times 10^{-4}$ \\
0.9 & $8.5 \times 10^{-13}$ & $4.1 \times 10^{-15}$ & $6.2 \times 10^{-4}$ \\
1.0 & $5.0 \times 10^{-11}$ & $8.3 \times 10^{-13}$ & $1.5 \times 10^{-4}$ \\
\hline \hline
\end{tabular}

with the boundary conditions $\mathrm{y}(0)=0$ and $\mathrm{y}(1)=0$ and the exact solution $y(x)=x^{2}-x$. In here, $f(x)=\frac{2 x^{1 / 2}}{\Gamma(3 / 2)}+x^{2}-x, P(x)=0, R(x)=1$ and $S(x)=1$.

Given $n=51, p=3, h=0.2$, we calculate the estimator $\hat{y}\left(t_{i}\right) \mathrm{i}=1,2, \ldots, 51$ at point $t=t_{i}$,

$$
\begin{gathered}
\hat{y}\left(t_{0}\right)=e_{1}\left(\chi^{T} \varpi \chi\right)^{-1} \chi^{T} \varpi \Upsilon . \\
\chi=\left[\begin{array}{cccc}
1 & 0 & 0 & 0 \\
1 & -0.98 & 1.2796 & -1.8859 \\
1 & -0.96 & 1.373 & -2.2027 \\
1 & -0.94 & 1.4364 & -2.4226 \\
1 & -0.92 & 1.4847 & -2.5915 \\
\vdots & \vdots & \vdots & \vdots \\
1 & -0.08 & 2.171 & -2.5115 \\
1 & -0.06 & 2.1916 & -2.4508 \\
1 & -0.04 & 2.2128 & -2.3881 \\
1 & -0.02 & 2.2345 & -2.3234 \\
1 & 0 & 0 & 0
\end{array}\right]_{51 \times 4}
\end{gathered}
$$




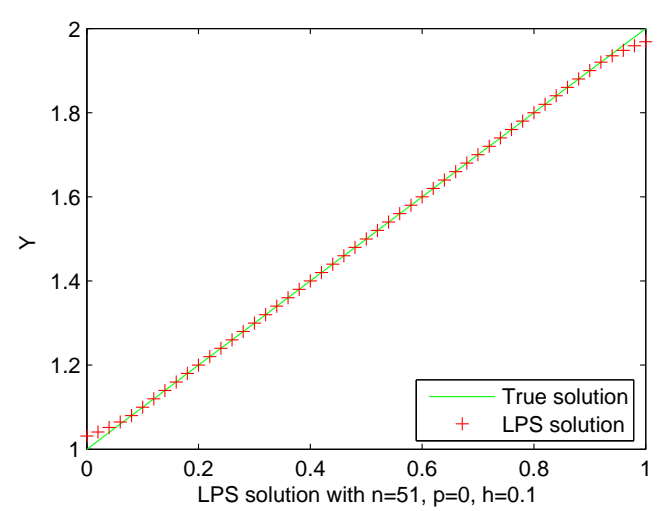

Fig. 1 LPS solution with $n=51, \mathrm{p}=0, \mathrm{~h}=0.1$ for example 1 .

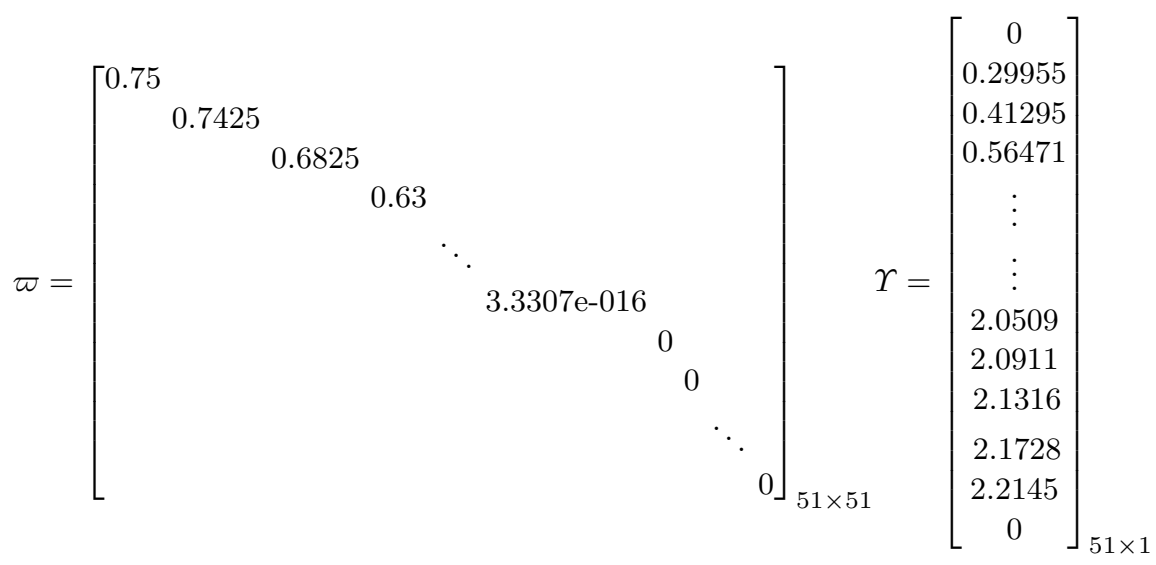

In fig 3, local polynomial smoother approximate analytical solution of example 2 with parameters $n=51, p=3, h=0.1$ and shows efficiency and high accuracy. 


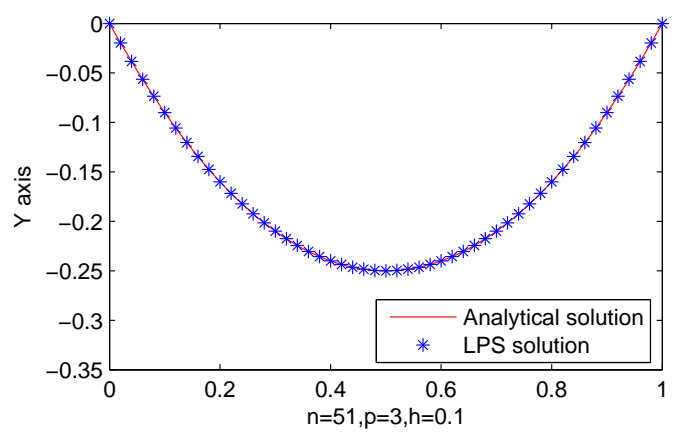

Fig. 2 LPS solution with $n=51, p=3, h=0.1$ for example 2 .

Table 2 Comparison of the absolutes for the numerical results and absolute errors at point $t_{i}=0.0: 0.2: 1.0$ for Example 2.

\begin{tabular}{cccc}
\hline \hline \multicolumn{1}{c}{$\begin{array}{c}\text { Absolute errors } \\
t_{i} \\
\text { for } n=51, p=3, h=0.2 \\
\text { LPS method }\end{array}$} & $\begin{array}{c}\text { Absolute errors } \\
\text { for } N=10 \alpha=0.5 \text { with the } \\
\text { pseudospectral method [33] }\end{array}$ & $\begin{array}{c}\text { Absolute errors } \\
\text { for } h=1 / 20 \alpha=0.5 \text { with } \\
\text { Adams-Bashforth method [34] }\end{array}$ \\
\hline \hline 0.0 & $1.05 \times 10^{-13}$ & $5.14 \times 10^{-5}$ & $3.63 \times 10^{-4}$ \\
0.2 & $7.46 \times 10^{-13}$ & $1.55 \times 10^{-4}$ & $4.56 \times 10^{-3}$ \\
0.4 & $6.38 \times 10^{-12}$ & $3.81 \times 10^{-4}$ & $6.10 \times 10^{-4}$ \\
0.6 & $3.27 \times 10^{-11}$ & $2.01 \times 10^{-5}$ & $6.19 \times 10^{-4}$ \\
0.8 & $9.10 \times 10^{-13}$ & $6.32 \times 10^{-4}$ & $1.33 \times 10^{-3}$ \\
1.0 & $7.26 \times 10^{-14}$ & $6.83 \times 10^{-4}$ & $3.42 \times 10^{-3}$ \\
\hline \hline
\end{tabular}

\section{Conclusions}

In this paper, we propose a fast algorithm based on local polynomial smoother which is applied for the numerical solution of Bagley-Torvik fractional differential equations (FDEs). The error result of LPS solution is very small and calculation processes are simple and feasible. Compared with Legendre operational matrix and pseudo-spectral method, LPS methods converges to solutions with fewer number of nodes and possess more accuracy. Moreover, it is more flexible to resolve problems just only adjusting 
parameters $p$, bandwidth $h$.

\section{References}

1. Bagley RL, Torvik PJ. On the appearance of the fractional derivative in the behavior of real materials. Journal of Applied Mechanics 1984; 51(2):294-298.

2. Bagley RL, Torvik PJ. Fractional calculus a different approach to the analysis of viscoelastically damped structures. The American Institute of Aeronautics and Astronautics $1983 ; 21(5): 741-748$.

3. K.B. Oldham, J. Spanier, The Fractional Calculus, Academic Press, New York, London, 1974.

4. Podlubny I. Fractional Differential Equations. Academic Press: San Diego, 1999.

5. Ray SS, Bera RK. Analytical solution of the Bagley-Torvik equation by Adomian decomposition method. Applied Mathematics and Computation 2005; 168(1):398-410.

6. S. Saha Ray, R.K. Bera, Analytical solution of the Bagley Torvik equation by Adomian decomposition method, Applied Mathematics and Computation 168 (2005) 398-410

7. Enesiz Y, Keskin Y, Kurnaz A. The solution of the Bagley-Torvik equation with the generalized Taylor collocation method. Journal of the Franklin Institute 2010; 347:452-466.

8. Diethelm K, Ford NJ. Numerical solution of the Bagley-Torvik Equation. Bit Numerical Mathematics 2002; 42(3):490-507.

9. K. Diethelm, N.J. Ford, A.D. Freed, A predictor-corrector approach for the numerical solution of fractional differential equations, Nonlinear Dyn. 29 (2002) 3-22.

10. S. Saha Ray, On Haar wavelet operational matrix of general order and its application for the numerical solution of fractional Bagley-Torvik equation, Applied Mathematics and Computation, 218 (2012) 5239-5248

11. Diethelm K. The analysis of fractional differential equations. Berlin: Springer-Verlag; 2010.

12. Diethelm K, Ford JM, Ford NJ, Weilbeer M. Pitfalls in fast numerical solvers for fractional differential equations. J Comput Appl Math 2006;186(2):482-503.

13. Ford NJ, Connolly JA. Comparison of numerical methods for fractional differential equations. Commun Pure Appl Anal 2006;5(2):289-307.

14. Luchko Y, Gorenflo R. An operational method for solving fractional differential equations with the Caputo derivatives. Acta Math Vietnam 1999;24(2):207-33.

15. Hashim I, Abdulaziz O, Momani S. Homotopy analysis method for fractional IVPs. Commun Nonlinear Sci Numer Simul 2009;14(3):674-84.

16. Arikoglu A, Ozkol I. Solution of fractional differential equations by using differential transform method. Chaos Solitons Fractals 2007;34(5):1473-81.

17. Odibat Z, Momani S, Erturk VS. Generalized differential transform method: application to differential equations of fractional order. Appl Math Comput 2008;197(2):467-77.

18. Erturk VS, Momani S, Odibat Z. Application of generalized differential transform method to multi-order fractional differential equations. Commun Nonlinear Sci Numer Simul 2008;13(8):1642-54.

19. Podlubny I. Matrix approach to discrete fractional calculus. Fract Calc Appl Anal $2000 ; 3(4): 359-86$. 
20. Deng W. Short memory principle and a predictor-corrector approach for fractional differential equations. J Comput Appl Math 2007;206(1):174-88.

21. Saadatmandi A, Dehghan M. A new operational matrix for solving fractional-order differential equations. Comput Math Appl 2010;59(3):1326-36.

22. Al-Mdallal QM, Syam MI, Anwar MN. A collocation-shooting method for solving fractional boundary value problems. Commun Nonlinear Sci Numer Simul 2010;15(12):3814-22.

23. Garrappa R, Popolizio M. On accurate product integration rules for linear fractional differential equations. J Comput Appl Math 2011;235(5):1085-97.

24. Liyun Su, Tianshun Yan. Numerical Solution of Integro-Differential Equations with Local Polynomial Regression, Open Journal of Statistics, 2012;2:352-355.

25. Liyun Su, Tianshun Yan. Local Polynomial Regression Solution for Partial Differential Equations with Initial and Boundary Values, Discrete Dynamics in Nature and Society, Volume 2012, Article ID 201678, 11 pages.

26. Liyun Su, Tianshun Yan. Local Polynomial Regression Solution for Differential Equations with Initial and Boundary Values, Mathematical Problems in Engineering, Volume 2013, Article ID 530932, 5 pages.

27. J. Fan, Q. Yao. Nonlinear Time Series: Nonparametric and Parametric Methods, Springer, New York, USA, 2003.

28. J. Fan, I. Gijbels. "Local Polynomial Modelling and Its Applications", Chapman Hall, London, UK, 1996.

29. J. Fan, I. Gijbels. Data-driven bandwidth selection in local polynomialfitting: variable bandwidth and spatial adaptation, Journal of the Royal Statistical Society, vol. 57, Series B, 371-394, 1995.

30. Liyun Su, Yanyong Zhao, Tianshun Yan. Two-stage Method Based on local Polynomial Fitting for a Linear Heteroscedastic Regression Model and Its Application in Economics , Discrete Dynamics in Nature and Society, Vol, 2012, Article ID 696927, 17 pages, 2012.

31. Jafari H, Yousefi SA, Firoozjaee MA, Momani S, Khalique CM. Application of Legendrewavelets for solving fractional differential Equations. Computers \&Mathematics with Applications, 2011;62:1038-1045.

32. Abbas Saadatmandi, Mehdi Dehghan. A new operational matrix for solving fractionalorder differential equations, Computers and Mathematics with Applications, 2010; 59 13261336 .

33. Esmaeili S, Shamsi M. A pseudo-spectral scheme for the approximate solution of a family of fractional differential equations. Communications in Nonlinear Science and Numerical Simulation, 2011; 16: 3646-3654.

34. Diethelm K, Ford NJ, Freed AD. Detailed error analysis for a fractional Adams method.Numerical Algorithms, 2004; 36(1): 31-52.

(C) 2016 by the authors; licensee Preprints, Basel, Switzerland. This article is an open access article distributed under the terms and conditions of the Creative Commons by Attribution (CC-BY) license (http://creativecommons.org/licenses/by/4.0/). 